\title{
Perspectivas del bambú para la construcción en México
}

\author{
Victor Rubén Ordóñez Candelaria ${ }^{1}$
}

\section{RESUMEN}

Este documento presenta un panorama actual sobre el uso del bambú en México y las perspectivas que se tienen de este material, sin que el análisis sea en toda su amplitud, ya que se restringe principalmente al uso de este material en la construcción. Se ha realizado una investigación bibliográfica sobre el uso del bambú en el mundo y en México; se han encontrado cosas muy interesantes que permiten ver el potencial del mismo en diferentes aplicaciones. Se incrementa el interés sobre esta especie cuando se sabe que su crecimiento es sorprendente y que pueden obtenerse cosechas del producto en lapsos de 5 a 6 años, a diferencia dela mayoría de especies maderables que requieren de un tiempo cuatro veces mayor para alcanzar su madurez. En cuanto a sus propiedades mecánicas presenta una gran ventaja sobre algunas maderas que se utilizan regularmente en la construcción.

PALABRAS CLAVE:

Bambú, propiedades físicas y mecánicas, preservación, método "Boucherie".

\section{ANTECEDENTES}

En Asia el bambú representa un recurso muy importante para la economía de varios países; de los 10 millones de toneladas que se producen anualmente en

\section{ABSTRACT}

In this paper a review about the current use of bamboo use Mexico is presented along with the prospects of this material in the construction industry mainly. A bibliographic review of bamboo in the world and Mexico was made, and some interesting points that increase its potential for several aplications were found. Their extraordinary growth which could be about 5-6 years to reach a mature state, as oppsed to most wood species, which takes about four times as much, heightens the interest in this plant. In regards to the mechanical and physical properties, bamboo has advantages over several wood species which are used regularly in building construction.

KEY WORDS:

Bamboo, physical and mechanical properties, preservation, "Boucherie" method.

el mundo, la mayor parte se producen en esa región. Solamente en China se estima que el crecimiento de los bosques de bambú, anualmente es de 3.5 millones de toneladas (Sharma, 1980). 
Su utilización cubre un rango muy amplio de aplicaciones como son: artesanías y pulpa para papel; en la India 80 de sus fábricas de papel dependen casi totalmente del bambú. Filipinas utiliza el $80 \%$ de sus recursos de bambú en la construcción y en aplicaciones rurales, y en muchas partes del mundo los brotes de algunas especies se utilizan como alimento humano.

El uso del bambú en la construcción, principalmente de vivienda es muy amplio en algunos países del continente americano. En países como Colombia y Costa Rica se llevan a cabo programas de investigación y desarrollo de viviendas con este material. Su aplicación se realiza de maneras muy diferentes, desde la construcción de armaduras para cubiertas con las cañas de bambú completas cortadas solamente a la longitud requerida por diseño, hasta las cañas cortadas en tiras para fabricar paneles para muros de viviendas, sobre los cuales se coloca una capa de mortero para cerrar los muros (Hidalgo, 1978).

Costa Rica cuenta desde 1996 con un Centro de Investigación y Tecnología Aplicada al Bambú que tiene como objetivo la investigación, la capacitación y la promoción del uso e industrialización del bambú. Este centro tiene como antecedente el proyecto nacional del bambú que tenía como propósito principal satisfacer la demanda de vivienda en el medio rural de bajos ingresos utilizando un material diferente como es el bambú.

En los bosques naturales de bambú en la India, en general, se llegan a tener rendimientos de 2.5 a 4.0 ton/ha, y con un manejo forestal conveniente su producción se eleva hasta 6.0 a 7.5 ton/ha. Centrándonos en una revisión de la información disponible acerca del bambú como material para la construcción se le observan cualidades muy interesantes, principalmente sus propiedades mecánicas, las que superan a varios de los materiales comunes de la construcción (Tabla 1).

En esta información presentada por el Profesor Janssen (1980) se muestran valores de diseño de los materiales estructurales más comunes comparados con el bambú; aunque no especifica bajo que tipo de solicitación se determinaron los valores reportados, es evidente que los valores para concreto corresponden al material sin refuerzo. De otro estudio realizado por Janssen (1980) con la especie Bambusa blumeana, se presentan en la tabla 2 los valores de resistencia obtenidos con pequeñas probetas.

Mohmod et al. (1993) realizaron ensayos con la misma especie obteniendo valores de resistencia para material de 1, 2 y 3 años de edad y a diferentes alturas del tallo definidas como base (B), parte media (M) y extremo (E). En la tabla 3 se presentan los resultados de este estudio con respecto a la resistencia de esta especie de bambú, donde se observa que la resistencia varía con respecto a estos dos factores. Para completar la información y efectuar una revisión comparativa entre las propiedades de diferentes especies de bambú y de lugares diferentes se presentan, en la tabla 4, resultados de estudios efectuados en Colombia con la Guadua (Guadua angustifolia) en condición seca.

Revisando los valores de resistencia presentados en las tablas, se observa que el bambú tiene propiedades mecánicas muy altas con relación a la madera y aún con el concreto. Esto le da un potencial estructural excelente que poco se explota en el mundo, excepto por algunos países que tienen larga tradición en su uso como son India, Malasia, China, y apenas hace pocos años en el continente americano: en Colombia y Costa Rica se han iniciado estudios de gran alcance para utilizar sus recursos de bambú para satisfacer necesidades de vivienda así como de estructuras para otros fines. 
Tabla 1. Propiedades de diseño de diferentes materiales estructurales y del bambú

\begin{tabular}{||l|c|c|c|c|c||}
\hline MATERIAL & $\begin{array}{c}\text { RESISTENCIA } \\
\text { DE DISEÑO } \\
(\mathrm{R}) \\
\left(\mathrm{KG} / \mathrm{CM}^{2}\right)\end{array}$ & $\begin{array}{c}\text { MASA POR } \\
\text { VOLUMEN } \\
(\mathrm{M}) \\
\left(\mathrm{KG}^{2} \mathrm{M}^{3}\right)\end{array}$ & $\begin{array}{c}\text { RELACION DE } \\
\text { RESISTENCIA } \\
(\mathrm{R} / \mathrm{M})\end{array}$ & $\begin{array}{c}\text { MODULO DE } \\
\text { ELASTICIDAD } \\
(\mathrm{E}) \\
\left(\mathrm{KG} / \mathrm{CM}^{2}\right)\end{array}$ & $\begin{array}{c}\text { RELACION } \\
\text { DE RIGIDEZ } \\
(\mathrm{E} / \mathrm{M})\end{array}$ \\
\hline Concreto & 82 & 2400 & 0.032 & 127400 & 53 \\
Acero & 1630 & 7800 & 0.209 & 2140000 & 274 \\
Madera & 76 & 600 & 0.127 & 112000 & 187 \\
Bambú & 102 & 600 & 0.170 & 203900 & 340 \\
\hline
\end{tabular}

Tomado de: http://www.ingersoll-rand.com/compair/ap-may97/bamb-4.htm

Tabla 2. Propiedades mecánicas de Bambusa blumeana en condición seca

\begin{tabular}{||l|c||}
\hline Resistencia a compresión $\left(\mathrm{kg} / \mathrm{cm}^{2}\right)$ & 825 \\
\hline Resistencia a flexión $\left(\mathrm{kg} / \mathrm{cm}^{2}\right)$ & 856 \\
\hline Módulo de elasticidad $\left(\mathrm{kg} / \mathrm{cm}^{2}\right)$ & 203873 \\
\hline Resistencia en cortante paralelo a la fibra $\left(\mathrm{kg} / \mathrm{cm}^{2}\right)$ & 23 \\
\hline Resistencia a tensión $\left(\mathrm{kg} / \mathrm{cm}^{2}\right)$ & 2038 - 3 058 \\
\hline
\end{tabular}

Tomado de Janssen (1980).

Tabla 3. Promedio de las propiedades mecánicas de Bambusa blumeana, en condición verde

\begin{tabular}{|c|c|c|c|c|c|c|c|c|c|}
\hline \multirow{3}{*}{ PROPIEDAD } & \multicolumn{9}{|c|}{ EDAD } \\
\hline & \multicolumn{3}{|c|}{1 año } & \multicolumn{3}{|c|}{2 años } & \multicolumn{3}{|c|}{3 años } \\
\hline & $\mathrm{B}$ & $M$ & $E$ & $\mathrm{~B}$ & M & $E$ & $\mathrm{~B}$ & $M$ & $E$ \\
\hline Cortante $\left(\mathrm{kg} / \mathrm{cm}^{2}\right)$ & 41 & 46 & 47 & 44 & 47 & 48 & 47 & 50 & 51 \\
\hline $\begin{array}{l}\text { Compresión paralela } \\
\text { a la fibra }\left(\mathrm{kg} / \mathrm{cm}^{2}\right)\end{array}$ & 200 & 213 & 226 & 228 & 272 & 283 & 258 & 283 & 294 \\
\hline $\begin{array}{l}\text { Esfuerzo en el límite } \\
\text { de proporcionalidad }\left(\mathrm{kg} / \mathrm{cm}^{2}\right)\end{array}$ & 216 & 241 & 235 & 238 & 244 & 403 & 243 & 245 & 431 \\
\hline $\begin{array}{l}\text { Módulo de elasticidad } \\
\text { x } 1000\left(\mathrm{~kg} / \mathrm{cm}^{2}\right)\end{array}$ & 29 & 30 & 45 & 31 & 33 & 46 & 35 & 36 & 59 \\
\hline Módulo de ruptura $\left(\mathrm{kg} / \mathrm{cm}^{2}\right)$ & 1043 & 755 & 477 & 1345 & 931 & 638 & 1631 & 1141 & 757 \\
\hline
\end{tabular}

Tomado de: Mohmod, et al. (1993) 
Tabla 4. Propiedades mecánicas de la Guadua

\begin{tabular}{|c|c|c|c|c|c|c|c|c|c|c|}
\hline \multirow{3}{*}{ ESPECIE } & \multicolumn{4}{|c|}{$\begin{array}{l}\text { TENSION } \\
\mathrm{kg} / \mathrm{cm}^{2}\end{array}$} & \multicolumn{4}{|c|}{$\begin{array}{c}\text { COMPRESION } \\
\mathrm{kg} / \mathrm{cm}^{2}\end{array}$} & \multicolumn{2}{|c|}{$\begin{array}{c}\text { MODULO DE } \\
\text { ELASTICIDAD } \\
\mathrm{kg} / \mathrm{cm}^{2}\end{array}$} \\
\hline & \multicolumn{2}{|c|}{ sin nudo } & \multicolumn{2}{|c|}{ con nudo } & \multicolumn{2}{|c|}{ sin nudo } & \multicolumn{2}{|c|}{ con nudo } & & \\
\hline & mín. & máx. & mín. & máx. & mín. & máx. & mín. & máx. & mín. & máx. \\
\hline Guadua macana & 970 & 1659 & 943 & 1429 & 606 & 689 & 525 & 660 & -- & -- \\
\hline Guadua de Castilla & 1020 & 1560 & 548 & 1045 & -- & -- & -- & -- & 107000 & 173000 \\
\hline
\end{tabular}

Tomados de Hidalgo (1978).

\section{EL BAMBU EN MEXICO}

El uso del bambú en México se encuentra restringido principalmente a la fabricación de muebles y construcciones rurales cercanas a la zona donde crece esta especie. La distribución geográfica de los bambúes se presenta básicamente en las regiones tropicales y los estados con mayor número de especies son Chiapas, Veracruz y Tabasco. También en los estados de Jalisco y Michoacán se presenta un número importante de especies. La especie Olmeca recta es una especie endémica que no crece a alturas mayores a $200 \mathrm{msnm}$, en los estados de Oaxaca y Veracruz; la Otatea acuminata que crece en suelos calcáreos y clima seco y Alonemia clarkie que se distribuye en el estado de Chiapas.

Actualmente se están desarrollando en algunas partes de los estados de Veracruz, Tabasco y Chiapas plantaciones con especies introducidas de Asia y Sudamérica, entre las que se pueden mencionar por su extensión e importancia las de Agroindustria Moderna S.A., con plantaciones de Guadua en Tabasco y las de Cementos Apasco en Orizaba, Ver. también con especies de Guadua y del bambú conocido como "Plumoso" y "Africano", comúnmente.
Esto nos permite vislumbrar que en México se puede desarrollar el uso del bambú con muy buenas perspectivas, y que puede servirnos para aminorar el déficit de vivienda, en un principio en las zonas cercanas a donde crece el bambú.

La información tecnológica sobre las propiedades físicas y mecánicas del bambú en México prácticamente no existe. Por lo tanto es necesario desarrollar trabajos de investigación con el fin de subsanar estas deficiencias de información sobre las especies que crecen en México. Un trabajo de importancia y de gran utilidad consiste en clasificar los bambúes en grupos de acuerdo con su potencial aplicación (muebles, construcción, tableros, papel, etc.); por ejemplo, aquellas especies con propiedades mecánicas más altas se recomendarían para la construcción de estructuras.

Es conveniente, por lo tanto realizar este tipo de trabajos de investigación básica, además de los relativos a pruebas de elementos estructurales como muros, sistemas de cubierta y armaduras, campo en el cual tendría un impacto importante. Generar información en el área de la construcción les daría elementos a los dueños del recurso para obtener mayores beneficios económicos. 


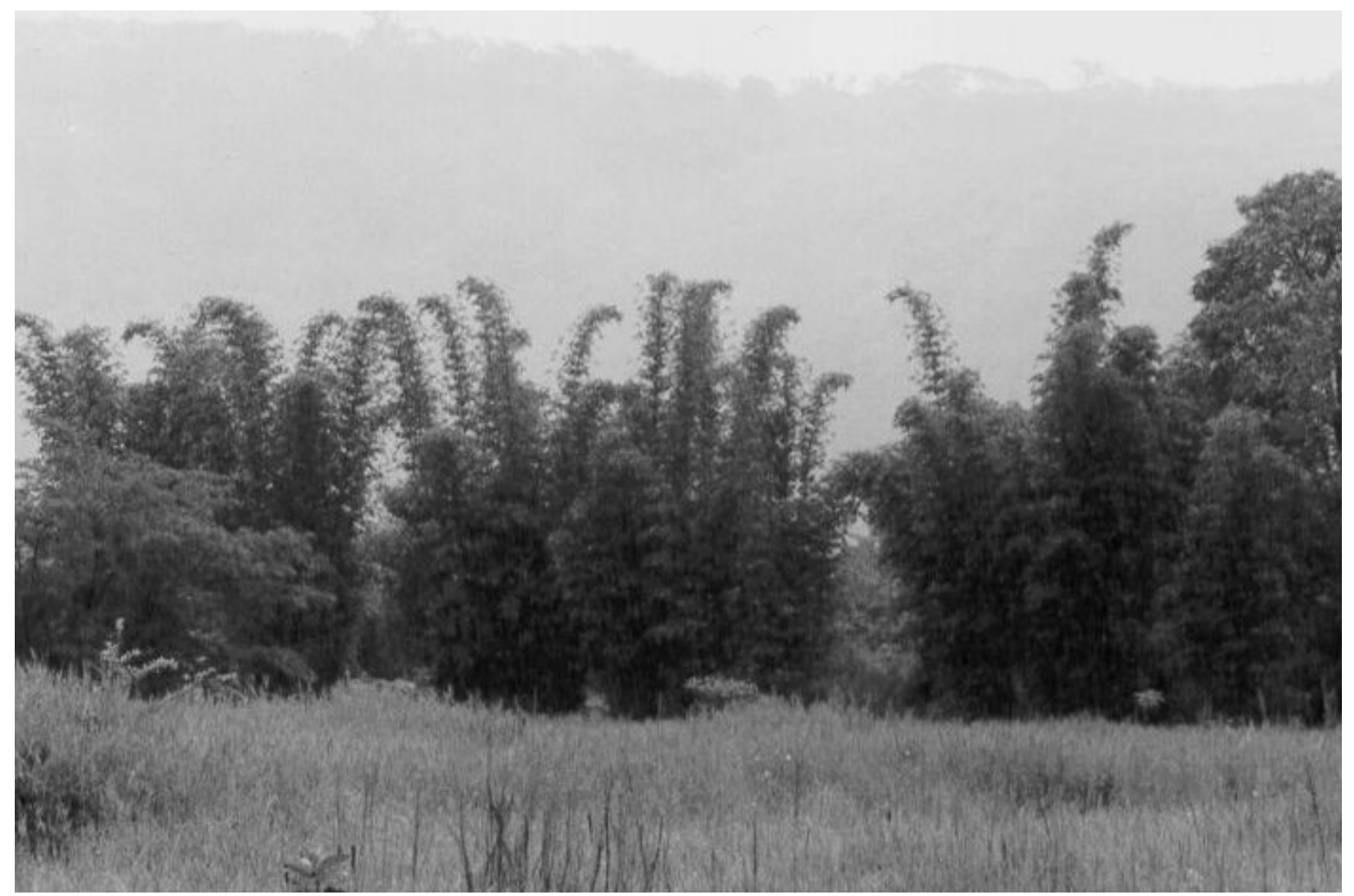

Plantación de bambú en las canteras de Cementos Apasco en Orizaba, Ver.

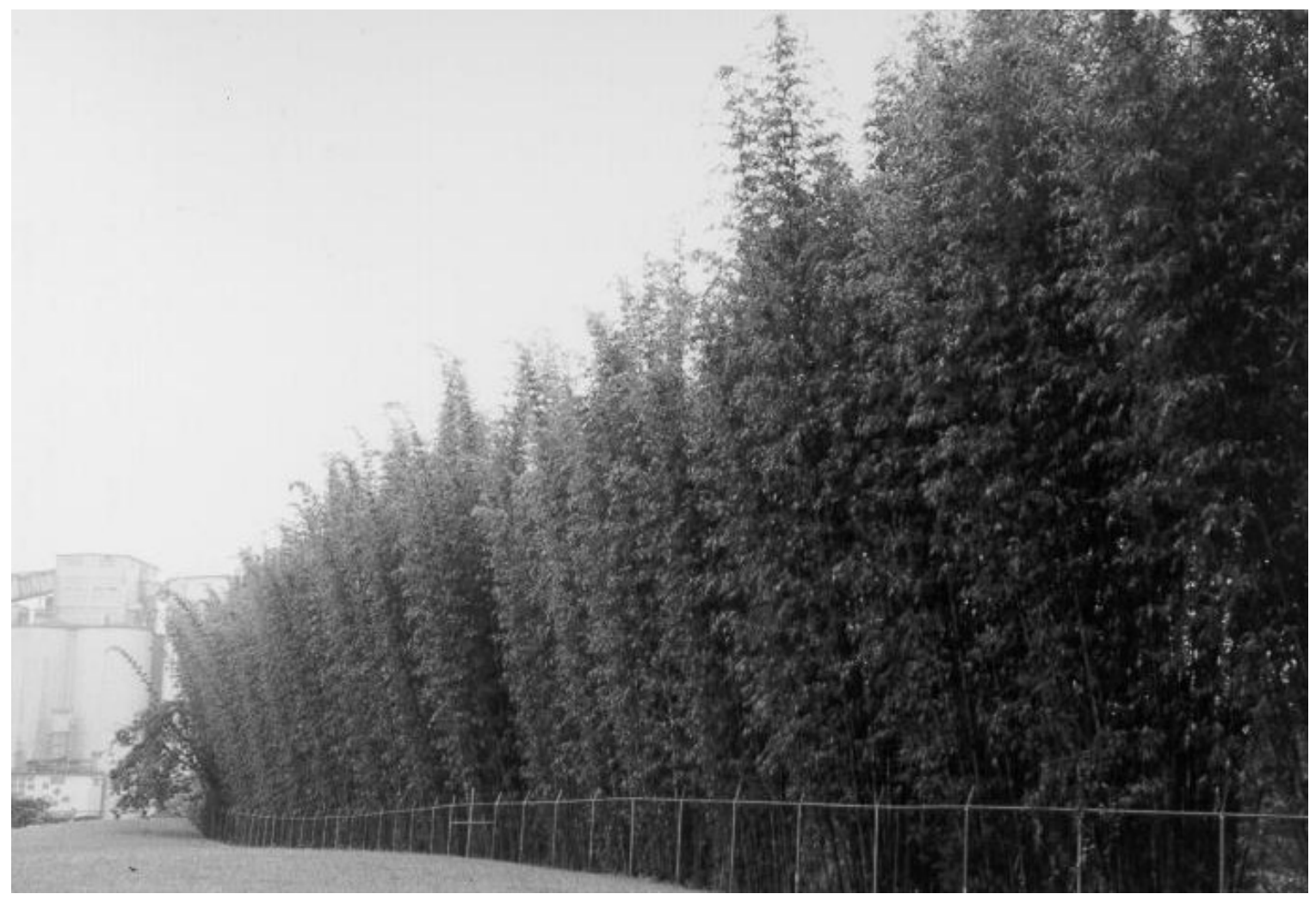

Plantación de bambú en la planta de Cementos Apasco en Orizaba, Ver 


\section{PROPUESTA DE PROYECTOS}

En este punto se proponen una serie de actividades en diferentes temas sobre los que se ha detectado un carencia de información a nivel nacional, pero son básicamente los de mayor importancia para usar el bambú como material de construcción.

Identificación de las especies. Se propone identificar las especies que nativas de México así como las introducidas, inicialmente de aquellas que tengan uso potencial en la construcción o para otros usos (muebles, papel, etc.), y de las plantaciones cuando así lo soliciten los dueños de las mismas.

Cada vez que se vaya a estudiar una especie para determinar sus propiedades físicas y mecánicas, la colecta incluirá la toma de ejemplares de herbario así como muestras para xiloteca y datos geográficos y climáticos de la zona donde crece. Para su mayor conocimiento un punto importante para seleccionar el material por colectar es que el mismo haya llegado a su madurez ya que la investigación bibliográfica indica que las propiedades mecánicas del bambú son más altas en individuos maduros, en promedio a la edad de 5 años.

Caracterización tecnológica de las especies. La mayor carencia de información se da precisamente sobre las propiedades físicas y mecánicas en los bambúes de México. Igualmente se requieren trabajos de biodeterioro $y$ preservación para incrementar su durabilidad. Es recomendable determinar los datos básicos, las propiedades por estudiar para obtener una caracterización lo más completa serían las siguientes:

Propiedades Físicas:

Densidad Básica

Contracciones

Secado

Contenido de humedad
Propiedades Mecánicas:

Flexión estática

Compresión paralela a la fibra

Tensión en pequeñas probetas

Cortante paralelo a la fibra

Impacto

Dureza "Janka"

Extracción de clavos

Para efectuar las pruebas de la lista citada se tratarán de ajustar las pruebas de las normas ASTM D 143-94 para pequeñas probetas de madera o con alguna de las normas que se utilizan en el continente asiático.

Sistema de tratamiento para preservación del bambú. Como el bambú es muy susceptible al ataque de los insectos y de los hongos, lo más conveniente es aplicarle algún tratamiento con preservadores para incrementar su vida útil bajo cualquier ambiente. Tamolang et al. (1980) han observado que los almidones del bambú contribuyen a su susceptibilidad a los insectos; se recomienda usar cañas de bambú maduro (más de tres años de edad). Bajo condiciones normales, la vida útil del bambú es de 1 a 3 años en contacto con el suelo; en interiores se incrementa su vida útil hasta 4-7 años y en zonas rurales de la India se ha encontrado que para el bambú usado en cocinas expuesto al humo y otras substancias la vida útil llega a ser de 10 a 15 años. Finalmente en condiciones marinas sólo llega a tener una vida de 6 meses.

Para el tratamiento preservador del bambú Grover (1957) y Liese (1980) recomiendan un método rápido y eficiente, el Método Modificado de Boucherie; a grandes rasgos este método consiste en substituir la savia de la caña con una solución preservadora; esto obliga a aplicar el tratamiento a las cañas recién cortadas antes de que pierdan el agua que contienen. 


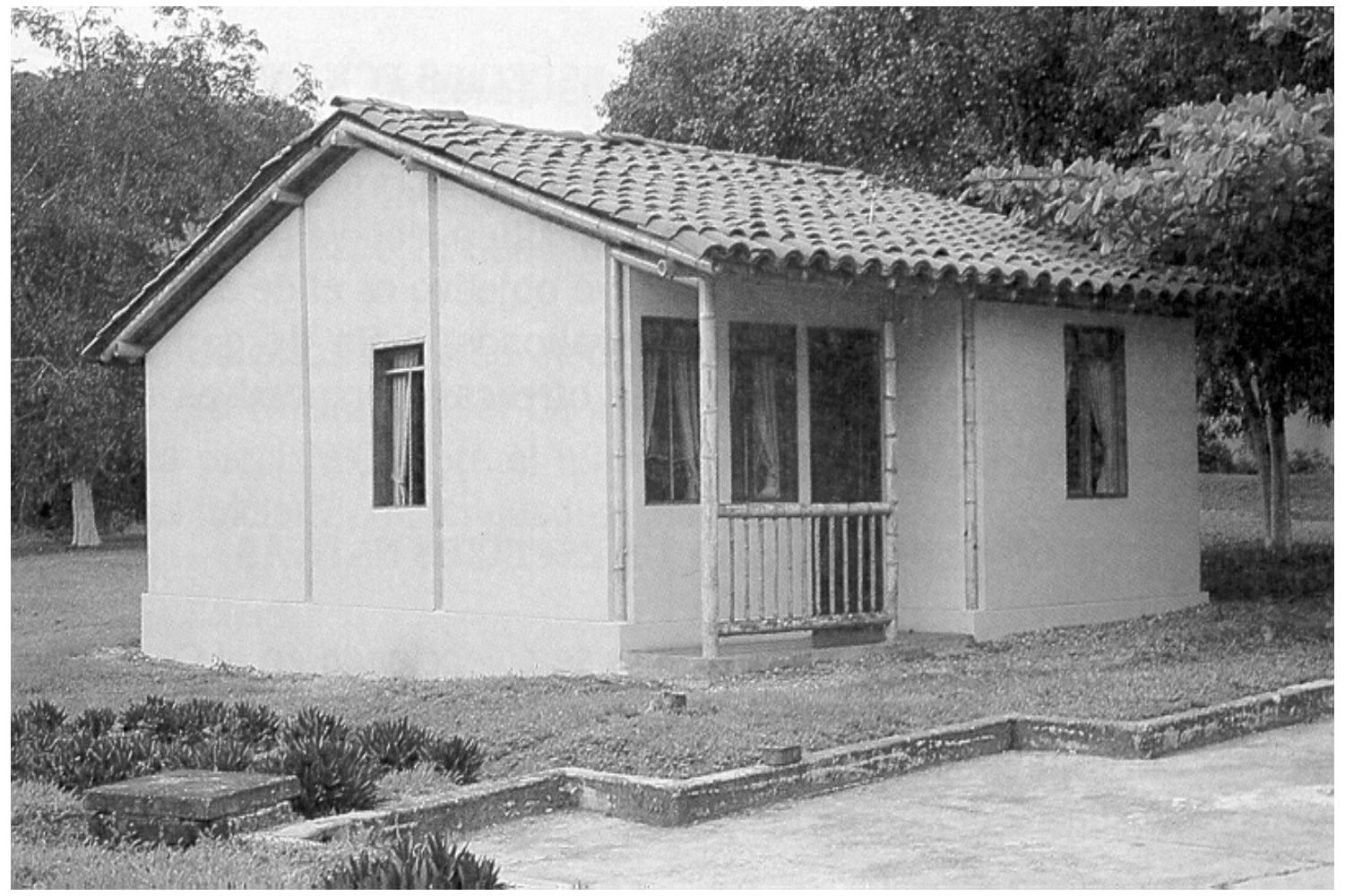

Construcción de casas de interés social con bambú.

(Tomada de Cruz, 1994)

Sistemas constructivos para vivienda. El uso más interesante y de mayor impacto del bambú se puede tener en la construcción de viviendas. Considerando que el déficit de vivienda en México es muy grande, ésta puede ser una alternativa para abatirlo a bajo costo en un sector muy amplio de la población. Es conveniente que se desarrollen algunos sistemas de construcción con el bambú para lograr este objetivo.

Con el fin de realizar un diseño estructural confiable de los elementos para la construcción de una vivienda se deberán ensayar prototipos de sistemas estructurales de la construcción como son: muros, entrepisos y techos, sometiéndose a cargas similares a las que se ven sujetos durante su vida en servicio, por ejemplo:
Muros:

Cargas de compresión

Cargas laterales horizontales

Cargas uniformes

Entrepisos y Techos:

Cargas uniformes

Cargas concentradas estáticas

Cargas de impacto

Investigaciones en elementos estructurales. Para aplicaciones en edificio mayores que una vivienda, como bodegas y naves industriales o semejantes, se requieren otros tipos de pruebas, como resistencia de uniones estructurales, propiedades mecánicas en flexión, compresión y tensión con elementos de tamaño real, comportamiento de armaduras, etc. Con la información que se genere en este tema se tendrá la posibilidad de crear estructuras seguras y eficientes con este material. 


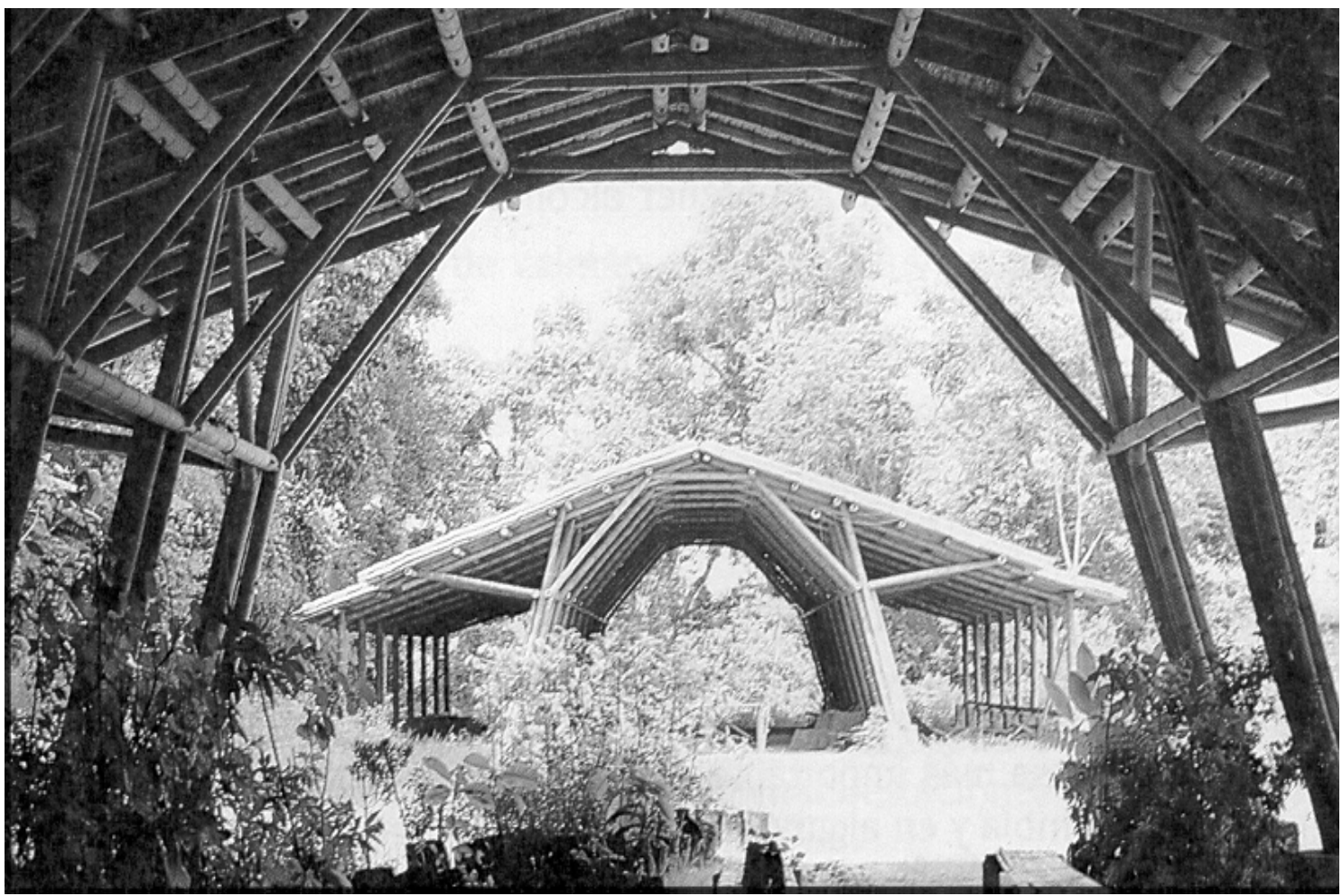

Construcción tipo industrial con bambú.

(Tomada de Cruz, 1994)

\section{COMENTARIOS FINALES}

El bambú se presenta como un recurso con algunas ventajas sobre varias especies maderables en cuanto a propiedades mecánicas. Otra gran ventaja es su crecimiento superior a cualquier especie maderable ya que alcanza su madurez en un tiempo de 5 a 6 años (Vela,1982), y su rendimiento por hectárea es similar o mayor que el de algunas especies maderables.

Por las perspectivas que tiene este recurso, se requiere impulsar plantaciones ya que tienen grandes posibilidades de éxito en varias regiones del país. Finalmente, debido a la falta de información sobre sus propiedades tecnológicas, es importante que se incrementen este tipo de estudios y se elaboren manuales de construcción, manuales de diseño así como un sistema de clasificación de las especies de bambú.
Las plantaciones de bambú a las extensiones de crecimiento natural de estas especies generan efectos benéficos en el suelo como evitar o detener la erosión del mismo e incrementa la retención de agua en el subsuelo por su sistema de raíces.

Precisamente las plantaciones de bambú de Cementos Apasco tienen como objetivo la restauración o recuperación del suelo en la zona donde la explotación de la cantera de calizas por la planta de cementos ha dejado un suelo sin materia orgánica. La importancia que tiene el impulsar las plantaciones de bambú y realizar su aprovechamiento sustentable, estriba por un lado en los beneficios ecológicos y por otro, se cuentan los beneficios económicos del aprovechamiento del bambú para su utilización en diversas aplicaciones. 


\section{LITERATURA CONSULTADA}

Cruz, R.H. 1994. La Guadua: Nuestro bambú. Corporación Autónoma Regional del Quindio. Centro Nacional para el Estudio del Bambú-Guadua. Colombia. 293 p.

Espiloy, Z.B. 1971. Some properties and uses of bamboos. Technical Note No. 109, Forest Products Research and Industries Development Comission. National Science Development Board College. Filipinas.

Grover, P.N. 1957. Preservation of bamboos by modified Boucherie process. Journal of Timber Dryers. Vol. III(3):16-23.

Hidalgo, L.O. 1978. Nuevas técnicas de construcción con bambú. Estudios Técnicos Colombianos Ltda. Universidad Nacional de Colombia. Bogotá, Colombia. p:26-137.

Janssen, J.J.A. 1980. The mechanical properties of bamboo used in construction. Eindhoven University of Technology, Netherlands. In: Proceedings: Bamboo Research in Asia. IDRC- IUFRO. p:173-188.

Janssen, J.J.A. 1981. The relationship between the mechanical properties and biological and chemical composition of bamboo. Eindhoven University of Technology, Netherland. Proceedings of Congress Group 5.3. Production and Utilization of Bamboo and Related Species. XVII IUFRO World Congress. Kyoto, Japan. p:2732.

Jayanetti, D.L. 1975. Some methods of preservative treatment of bamboo. The Sri Lanka Forester, Vol. 12(2):101-103.

Liese, W. 1980. Preservation of bamboos. Federal Research Center for Forestry and Forest Products.
Hamburk-Reinbek, W. Germany. In: Proceedings: Bamboo Research in Asia. IDRC-IUFRO. p:165-172.

Liese, W. 1986. Characterization and utilization of bamboo. University of Hamburg, FRG. In: Proceedings of project group $P$ 5.04. Production and utilization of bamboo and related Species. XVIII IUFRO World Congress. Ljubljana, Yugoslavia. p:1116.

Mejía S., Ma. T. y G. Castillo C. 1987. El Bambú. INIREB Informa. Comunicado No. 81. Instituto Nacionalde Investigaciones sobre Recursos Bióticos. Xalapa, Ver. México. 4 p.

McClure. 1953. Bamboo as a building construction material. Foreign Agricultural Service, U.S. Dept. of Agriculture, Washington, D.C. EUA. 52 p.

Mohmod, A.L.., Amin, A.H., Kasim, J. y Jusuh, M.Z. 1993. Effects of anatomical characteristics on the physical and mechanical properties of Bambusa blumeana. Journal of Tropical Forest Science 6(2):159-170. Forest Research Institute of Malaysia.

Purushotham, A., S.K. Sudan y V. Sagar. 1953. Preservative treatement of green bamboos under low pnewmatic pressures. Indian Forester. Wood Preservation Branch, Forest Research Institute, Dehra Dun, India. Vol. 79(12):652-672.

Purushotham, A. 1963. A preliminary note on some experiments using bamboo as reinforcement in cement concrete. Journal of Timber and Dryers and Preserver's Association of India. Vol IX(4): p:3-14.

Sharma, Y.M.L. 1980. Bamboo in AsiaPacific region. Bamboo research in Asia: Proceedings of a workshop 
heldin Singapore. Ottawa, Ont. Canadá. IDRC. p:99-120.

Sonti, V.R., R.N. Karmakar y K. Dey. 1983. Low cost dome structure using CCA treated bamboos. Timber Development Association. India. Vol XXIX(4):35-40.

Tamolang, F.N., F.R. Lopez, J.A. Semana, R.F. Casin y Z.B. Espiloy. 1980. Properties and Utilization of Philippine
Erect Bamboo. FORPRIDE Digest Vol. IX(3) y (4). Filipinas. In: Proceedings: Bamboo research in Asia. IDRCIUFRO. p:189-200.

Tewari, M.C. y B. Singhj. 1979. Bamboos their utilization and protection against biodeterioration. Journal of Timber Development Association of India. Vol. $\mathrm{XXV}(4): 12-23$

Vela G., L. 1982. Los bambúes. Boletín Técnico No. 50. INIF-SARH. México, D.F. $38 p$ 